\title{
On the Exact Shape of the Horizontal Profile of a Topographically Rectified Tidal Flow
}

\author{
L. R. M. MAAS and J. T. F. ZIMMERMAN
}

Netherlands Institute of Sea Research, P.O. Box 59, 1790 AB Texel and Institute of Meteorology and Oceanography, University of Utrecht, The Netherlands

and

\section{N. M. TEMME}

Centre for Mathematics and Computer Science, P.O. Box 4079, 1009 AB Amsterdam, The Netherlands

(Received June 13, 1986; in final form August 1, 1986)

Starting from the nonlinear shallow water equations of a homogeneous rotating fluid we derive the equation describing the evolution of vorticity by a fluctuating bottom topography of small amplitude, using a multiple scale expansion in a small parameter, which is the topographic length scale relative to the tidal wave length. The exact response functions of residual vorticity for a sinusoidal bottom topography are compared with those obtained by a primitive perturbation series and by harmonic truncation, showing the former to be invalid for small topographic length scales and the latter to be only a fair approximation for vorticity produced by planetary vortex stretching. In deriving the exact shape of the horizontal residual velocity profile at a step-like break in the bottom topography, it is shown that the Lagrangian profile only exists in a strip having the width of the amplitude of the tidal excursion at both sides of the break, and that it vanishes outside that interval. Moreover, in the limit of small amplitude topography at least, it vanishes altogether for the generation mechanism by means of planetary vortex stretching. The Eulerian profile is shown to extend over twice the interval of the Lagrangian profile both for production by vortex stretching 
and by differential bottom friction. These finite intervals over which the residual velocity profiles exist for a step-like topography are not reproduced by harmonic truncation of the basic equation. This method gives exponentially decaying profiles, indicating spurious horizontal diffusion of vorticity. In terms of orders of magnitude, the method of harmonic truncation is reliable for residual velocity produced by vortex stretching but it overestimates the residual velocity produced by differential bottom friction by a factor 2 .

KEY WORDS: Coriolis force, inertio-gravity waves, topographic rectification, ultraviolet catastrophe, weak non-linearities.

\section{INTRODUCTION}

It is now an established fact, theoretically, that whenever a long inertio-gravity (tidal) wave propagates over a depth-varying sea bed, the horizontal length scale of which is much smaller than the wave length, a rectified current is produced, along with higher harmonics of the basic frequency. All two-dimensional studies of this nonlinear problem give the same qualitative conclusions; see recent reviews by Huthnance (1981), Zimmerman (1981a) and Robinson (1983). However, they may differ at some points considerably in quantitative details, probably due to their different handling of the nonlinear interactions.

Huthnance (1973), in the first study of topographic rectification, used the approach of a primitive perturbation series, appropriate for weak-nonlinearities, showing the residual current speed to be proportional to the slope of the bottom. This evidently implies an "ultraviolet catastrophe" if the bottom topographic length scale becomes very small (the slope going to infinity), an indication of the invalidity of the primitive series for stronger nonlinearities, although the horizontally integrated mass-transport given by Huthnance (1973) remains finite for small topographic length scales. Loder (1980) improved the theory by including feed back of the generated residual currents to the tidal velocity field. This method, which is in fact not a perturbation approach but a harmonic truncation in the frequency domain, surprisingly removes the catastrophic behaviour of the response for small topographic length scales, leading to results that seem to be uniformly valid for all strengths of the nonlinear interactions concerned. Particularly for a step-profile of the bottom topography, Loder (1980) shows that the rectified flow speed keeps a 
finite value and decays exponentially away from the step. Unfortunately, Loder's (1980) theory gives analytical results only for such a step-like profile. Zimmerman $(1978,1980)$ circumvents the problems of either the primitive perturbation series or the method of harmonic truncation by dealing with a "small-amplitude topography". At this expense, his results are exact and, moreover, they can be used to construct horizontal velocity profiles for any shape of a topography, the step-topography included. Being exact in this sense, these results can therefore be compared to the approximate results of Loder (1980), obtained by harmonic truncation (transforming these to the small-topographic limit, of course). It then appears that although Loder's (1980) response for a sinusoidal small-amplitude topography can be a fair approximation to the exact response as obtained by Zimmerman $(1978,1980)$, the final result for a steptopography differs in an important property: whereas Loder's (1980) rectified jet decays exponentially away from the step, the exact results we present here prove that, in the absence of viscosity, the jet width is two times the tidal excursion amplitude. This means that the method of harmonic truncation may give an erroneous impression of the jet width, as has already been inferred by Young (1983), discussing the moments of the residual vorticity distribution. That is, the first moment of the vorticity distribution around a steptopography represents the laterally integrated along-isobath residual transport, which is well-behaved, even in a primitive perturbation expansion (Huthnance, 1973), as is the second moment of the vorticity distribution, which represents the center of mass of the rectified flow, located exactly at the position of the steepest slope in the limit of small amplitude topography. However, for the third moment of the vorticity distribution, representing the width of the rectified flow, Young (1983) shows that this property may be overestimated by $60 \%$ using Loder's (1980) harmonic truncation method in the limit of a small step-width; whereas the primitive perturbation series evidently underestimates the jet width in that limit.

Therefore, we derive here the exact profile of both the Eulerian and the Lagrangian residual velocity at a step-like break in the topography. The final result for the Eulerian residual velocity distribution is a rather cumbersome expression in terms of (integrals over) special functions. These are certainly not of much practical use, 
but our purpose is only to confront the properties of the exact solution with those obtained by the method of harmonic truncation, which is practically much more attractive, because of the simple vorticity transfer functions that result from its application. Our results, however, show that care should be taken in using this approximation method.

The outline of our derivations is as follows. In the next section we give a formal derivation of the basic vorticity equation that was posed by Zimmerman $(1978,1980)$ in a more intuitive way. We recapitulate then his response functions for a sinusoidal topography in Section 3, and compare these to the result of primitive perturbation and harmonic truncation. Finally, these results are used to obtain the exact shape of the residual current velocity profile at a step in the bottom topography, which is the subject of Section 4 .

\section{BASIC EQUATION}

We start from the two-dimensional shallow water equations for a homogeneous fluid in a uniformly rotating medium

$$
\begin{gathered}
\partial \mathbf{u} / \partial t+(\mathbf{u} \cdot \nabla) \mathbf{u}+f \mathbf{n} \times \mathbf{u}+(D+\zeta)^{-1} r \mathbf{u}=-g \nabla \zeta+\mu \nabla^{2} \mathbf{u}, \\
\partial \zeta / \partial t+\nabla \cdot\{(D+\zeta) \mathbf{u}\}=0
\end{gathered}
$$

Here $\mathbf{u}$ is the two-dimensional horizontal velocity vector and $\zeta$ is the disturbance of sea level from its equilibrium position. Friction is represented by a linear friction coefficient $r$ parametrizing vertical momentum transfer and by a horizontal eddy viscosity $\mu$. The Coriolis parameter is denoted by $f$ and the acceleration of gravity by $g ; \mathbf{n}$ is the vertical unit vector pointing upwards.

In order to derive from the fully nonlinear equations (1) and (2) an approximate equation for the topographically generated vorticity by a tidal flow, it is essential to distinguish between three different "small parameters". The first one is related to the tidal wave proper. Let $U$ be a characteristic velocity scale of the wave, $Z$ a scale of the vertical displacement of the water surface, $\sigma$ the frequency, $L_{0}$ the wave-length and $H$ a depth scale. Then in order for the wave to be quasi-linear we must assume

$$
\varepsilon=Z / H=2 \pi U / \sigma L_{0}=2 \pi l_{0} / L_{0} \ll 1,
$$


where $l_{0}$ is the tidal excursion amplitude. The topography now introduces two further small parameters. As we deal with a "small amplitude" topography we must assume

$$
\delta=h / H \ll 1
$$

where $h$ is a characteristic scale of the variations in depth. These variations are assumed to have a characteristic horizontal scale, $L$, that is much smaller than the wave-length, $L_{0}$, so that finally

$$
\lambda=L / L_{0} \ll 1
$$

In order to expand in only one of the three parameters $\varepsilon, \delta$ and $\lambda$, we have to make a choice about their relative strength. As we are primarily interested in topographic length scales, $L$, of the order of the tidal excursion amplitude, $l_{0}$, we set

$$
\varepsilon=\lambda=\delta
$$

where the last equality is just an arbitrary choice. Having now two horizontal length scales over which variables may vary ( $L$ and $L_{0}$, $\left.L / L_{0} \ll 1\right)$ it seems appropriate to apply a multiple scale expansion of (1) and (2). Therefore we introduce the following scaling

$$
\begin{gathered}
\mathbf{u}=L \sigma \mathbf{u}^{\prime}, \quad \zeta=\varepsilon H \zeta^{\prime}, \quad t=t^{\prime} / \sigma, \quad \mathbf{x}=L \mathbf{x}^{\prime}+L_{0} \mathbf{X}^{\prime} \\
f=\sigma f^{\prime}, \quad r=\sigma H r^{\prime}, \quad \mu=\sigma L^{2} \mu^{\prime},
\end{gathered}
$$

where we have introduced the "fast" and "slow" horizontal coordinates $\mathbf{X}^{\prime}$ and $\mathbf{X}^{\prime}$. Then the $\boldsymbol{\nabla}$-operator reads

$$
\nabla=L^{-1}\left(\nabla^{\prime}+\lambda \tilde{\nabla}^{\prime}\right), \quad \lambda \ll 1
$$

where

$$
\nabla^{\prime}=\left(\partial / \partial x^{\prime}, \partial / \partial y^{\prime}\right) \quad \text { and } \quad \tilde{\nabla}^{\prime}=\left(\partial / \partial X^{\prime}, \partial / \partial Y^{\prime}\right)
$$

In its dimensionless form, the depth profile is then given by

$$
D^{\prime}\left(x^{\prime}\right)=1+\lambda h^{\prime}\left(x^{\prime}\right)
$$


assuming the depth only to vary over small length scales. Using (6), (7) and (8), and scaling (1) and (2) according to the given scheme, the dimensionless form of (1) and (2) (dropping primes), is

$$
\begin{gathered}
\lambda\left[\frac{\partial \mathbf{u}}{\partial t}+\{\mathbf{u} \cdot(\boldsymbol{\nabla}+\lambda \tilde{\mathbf{\nabla}})\} \mathbf{u}+f \mathbf{n} \times \mathbf{u}+\frac{r}{D+\lambda \zeta} \mathbf{u}\right] \\
=-(\boldsymbol{\nabla}+\lambda \tilde{\nabla}) \zeta+\lambda \mu(\nabla+\lambda \tilde{\nabla})^{2} \mathbf{u}, \\
\lambda \partial \zeta / \partial t+(\boldsymbol{\nabla}+\lambda \tilde{\boldsymbol{\nabla}}) \cdot\{D+\lambda \zeta) \mathbf{u}\}=0 .
\end{gathered}
$$

Expanding the dependent variables in a perturbation series

$$
\begin{aligned}
& \mathbf{u}=\mathbf{u}^{(0)}+\lambda \mathbf{u}^{(1)}+\lambda^{2} \mathbf{u}^{(2)}+\cdots, \\
& \zeta=\zeta^{(0)}+\lambda \zeta^{(1)}+\lambda^{2} \zeta^{(2)}+\cdots
\end{aligned}
$$

we have at zeroth order

$$
\nabla \zeta^{(0)}=\mathbf{0}, \quad \boldsymbol{\nabla} \cdot \mathbf{u}^{(0)}=0,
$$

hence

$$
\zeta^{(0)}=\zeta^{(0)}(\mathbf{X}, t), \quad \mathbf{u}^{(0)}=\mathbf{u}^{(0)}(\mathbf{X}, t)
$$

neglecting a divergenceless small scale velocity at this order as, up to this order, there is no forcing for such a field, whereas any preexisting field decays due to bottom friction. In first order, using (13) and (14), we find: $\nabla \zeta^{(1)}=\mathbf{0}$, hence

$$
\zeta^{(1)}=\zeta^{(1)}(\mathbf{X}, t)
$$

and $\boldsymbol{\nabla} \cdot\left(h \mathbf{u}^{(0)}+\mathbf{u}^{(1)}\right)=0$, hence

$$
\nabla \cdot \mathbf{u}^{(1)}=-\mathbf{u}^{(0)} \cdot \nabla h,
$$

for the "fast" scale, and for the "slow" scale

$$
\begin{gathered}
\partial \mathbf{u}^{(0)} / \partial t+f \mathbf{n} \times \mathbf{u}^{(0)}+r \mathbf{u}^{(0)}=-\tilde{\nabla} \zeta^{(0)}, \\
\partial \zeta^{(0)} / \partial t+\tilde{\nabla} \cdot \mathbf{u}^{(0)}=0
\end{gathered}
$$


TOPOGRAPHICALLY RECTIFIED TIDAL FLOW

which are the familiar linearized shallow water equations for a flatbottom sea. Up to this order, the small scale field has a rigid-lid, coupling the divergence of its velocity directly to the variations in depth. Its full dynamics appears at second order. Then we find for the small scale

$$
\partial \mathbf{u}^{(1)} / \partial t+\left(\mathbf{u}^{(0)} \cdot \nabla\right) \mathbf{u}^{(1)}+f \mathbf{n} \times \mathbf{u}^{(1)}+r \mathbf{u}^{(1)}-r \mathbf{u}^{(0)} h=-\nabla \zeta^{(2)}+\mu \nabla^{2} \mathbf{u}^{(1)},
$$

and an equation for the slow scale which is of no further interest here. Taking finally the curl of Eq. (19) by operating with the small scale $\nabla$, we arrive at

$$
\partial \eta / \partial t+\mathbf{u}^{(0)} \cdot \nabla \eta+r \eta-\mu \cdot \nabla^{2} \eta=f \mathbf{u}^{(0)} \cdot \nabla h-r\left(\mathbf{u}^{(0)} \times \nabla h\right) \cdot \mathbf{n},
$$

where

$$
\eta=\left(\nabla \times \mathbf{u}^{(1)}\right) \cdot \mathbf{n},
$$

and use has been made of (16). This is the vorticity equation for topographically induced perturbations by the basic tidal velocity $\mathbf{u}^{(0)}$ in the limit of small-amplitude topography, as discussed in Zimmerman $(1978,1980)$, which now has been given a formal justification by means of a multiple-scale expansion.

\section{SPECTRAL RESPONSE}

For given $\mathbf{u}^{(0)}(t)$, independent of the "fast" coordinate $\mathbf{x}$ we solve (20) by introducing the Fourier transformations

$$
\begin{aligned}
\eta(\mathbf{x}, t) & =\iint \hat{\eta}(\mathbf{k}, t) e^{-i \mathbf{k} \cdot \mathbf{x}} d \mathbf{k}, \\
h(\mathbf{x}) & =\iint \hat{h}(\mathbf{k}) e^{-i \mathbf{k} \cdot \mathbf{x}} d \mathbf{k},
\end{aligned}
$$

where the integrals are over all of the wave number space. Of course, for a specific topography, $h(\mathbf{x})$ is prescribed as well.

Let now $u^{(0)}$ be given as an elliptically polarized current

$$
\mathbf{u}^{(0)}=[\sin \sigma t, \pm \epsilon \cos \sigma t],
$$


where $\epsilon$ is the ellipticity, which should not be confused with the perturbation parameter introduced earlier. Transforming to polar coordinates such that

$$
\mathbf{k}=[k \cos \theta, k \sin \theta],
$$

(20) can be written as

$$
\begin{aligned}
& d \hat{\eta} / d t-\left(i k \sin t-r-\mu k^{2}\right) \hat{\eta} \\
& \quad=i \hat{h} k\left\{-f \sin t \pm r F(\theta, \epsilon) \cos \left(t \pm \alpha_{2} \pm\left(-\alpha_{1}\right)\right\}\right.
\end{aligned}
$$

where

$$
F(\theta, \epsilon)=\left\{\left[\left(1-\epsilon^{2}\right) \sin ^{2} \theta+\epsilon^{2}\right] /\left[\left(\epsilon^{2}-1\right) \sin ^{2} \theta+1\right]\right\}^{1 / 2},
$$

and

$$
\alpha_{1}=\tan ^{-1}(\epsilon \tan \theta), \quad \alpha_{2}=\tan ^{-1}\left(\epsilon^{-1} \tan \theta\right) .
$$

Both terms in the right-hand side of (26) still describe the two basic vorticity production mechanisms, viz. planetary vortex-stretching and differential bottom friction. Mathematically it is more convenient to regard the right-hand side of (26), as the sum of a contribution proportional to $\sin t$ and one proportional to $\cos t$, in which case, generally, both production mechanisms are mixed up depending on the values of $\alpha_{1}$ and $\alpha_{2}$. In the case of a circularly polarized velocity vector $\mathbf{u}^{(0)}$, however, we have a convenient mathematical and physical separation in the right-hand side of (26) since then

$$
\epsilon=1, \quad F(\theta, \epsilon)=1, \quad \alpha_{1}=\alpha_{2}=\theta .
$$

We also drop the distinction left and right oriented current in (24). Choosing in (24) the minus sign, we obtain for (26)

$$
d \hat{\eta} / d t-\left(i k \sin t-r-\mu k^{2}\right) \hat{\eta}=-i \hat{h} k(f \sin t+r \cos t) .
$$

It is easy to see that a solution of (30) can readily be transformed to one of the more general Eq. (26), so that from here on we pursue with the case of a circularly polarized "undisturbed" current velocity vector. We now solve for the non-transient solution of (30) in two steps. 


\subsection{Quasi-Lagrangian solution}

Equation (30) suggests the following transformation

$$
\hat{\eta}(k, t)=\hat{\omega}(k, t) e^{-i k \cos t} .
$$

Since this is equivalent to introducing a coordinate transformation

$$
\mathbf{x}^{\prime}=\mathbf{x}+\int \mathbf{u}^{(0)} d t
$$

in physical space [i.e. in Eq. (20)] we call $\hat{\omega}(k, t)$ the quasiLagrangian (Fourier transformed) vorticity, as it describes the vorticity seen by an observer moving with the "undisturbed" flow. Then $(30)$ reads

$$
d \hat{\omega} / d t+b(k) \hat{\omega}=a(k) e^{i k \cos t}\{f \sin t+r \cos t\},
$$

where

$$
a(k)=-i k \hat{h}, \quad b(k)=r+\mu k^{2} .
$$

Writing $\hat{\omega}^{(1)}$ for the vorticity driven by the term $\sin t$ of the nonhomogeneous term in (33) and $\hat{\omega}^{(2)}$ for the term cos $t$, employing the Fourier series

$$
e^{i k \cos t}=\sum_{n=-\infty}^{\infty} i^{n} J_{n}(k) e^{i n t}
$$

where $J_{n}$ is a Bessel function of the first kind of order $n$, the nontransient solutions for $\hat{\omega}^{(1)}$ and $\hat{\omega}^{(2)}$ read

$$
\begin{gathered}
\hat{\omega}^{(1)}=f a(k) \sum_{n=-\infty}^{\infty} \phi_{n}^{(1)}(k, b) e^{i n t}, \\
\phi_{n}^{(1)}(k, b)=-i^{n} n[b(k)+i n]^{-1} J_{n}(k) / k \\
\hat{\omega}^{(2)}=r a(k) \sum_{n=-\infty}^{\infty} \phi_{n}^{(2)}(k, b) e^{i n t} \\
\phi_{n}^{(2)}(k, b)=i^{n-1}[b(k)+i n]^{-1} J_{n}^{\prime}(k) .
\end{gathered}
$$


Note that in the quasi-Lagrangian vorticity field higher harmonics of the fundamental frequency are present. It can also be seen that for $n=0$, the residual vorticity field $\hat{\omega}^{(1)}$ vanishes whereas $\hat{\omega}^{(2)}$ will be different from zero. Hence topographic stretching of planetary vortex lines does not produce a Lagrangian residual current up to this order in $\delta=h / H$, any Lagrangian residual field being due to differential bottom friction. This is in accordance with Loder (1980) if his results are expanded in $\delta=h / H$, keeping only first order terms in $\delta$.

\subsection{Eulerian solution}

The Fourier transformed Eulerian vorticity field is obtained from (31), substituting (36)-(39). The general solution has been given in Zimmerman (1980). Here we shall only recapitulate the residual part, which reads

$$
\begin{gathered}
\hat{\eta}_{0}^{(1)}=i f a(k) \psi_{0}^{(1)}(k), \\
\psi_{0}^{(1)}(k)=2 k^{-1} \sum_{m=1}^{\infty} m^{2}\left(b^{2}+m^{2}\right)^{-1} J_{m}^{2}(k), \\
\hat{\eta}_{0}^{(2)}=\operatorname{ira}(k) \psi_{0}^{(2)}(k) \\
\psi_{0}^{(2)}(k)=b^{-1} J_{0}(k) J_{1}(k)-2 b \sum_{m=1}^{\infty}\left(b^{2}+m^{2}\right)^{-1} J_{m}(k) J_{m}^{\prime}(k) .
\end{gathered}
$$

Evidently the residual vorticity is proportional to the bottom slope times a wave number dependent weighting function $\psi_{0}^{(i)}$. In contrast to the quasi-Lagrangian solution (36)-(37), the Eulerian solution connected with topographic planetary vortex stretching, (40)-(41), now contains a nonvanishing residual component.

In order to compare the exact results, (40)-(43), with those obtained by approximation, we give here the asymptotic forms for $k \rightarrow 0$ and $k \rightarrow \infty$ of the weighting functions $\psi_{0}^{(i)}(k)$. An extensive discussion about asymptotics can be found in Temme and Zimmerman (1985), from which we quote

$$
\psi_{0}^{(1)}(k)=r \psi_{0}^{(2)}(k)=\frac{1}{2} k\left(1+r^{2}\right)^{-1}+O\left(k^{2}\right), \quad k \rightarrow 0 ;
$$




$$
\begin{gathered}
\text { TOPOGRAPHICALLY RECTIFIED TIDAL FLOW } \\
\psi_{0}^{(1)}(k)=\frac{1}{2} \mu^{-2} k^{-3}+O\left(k^{-5}\right), \\
\psi_{0}^{(2)}(k)=\frac{1}{2} \mu^{-2} k^{-5}+O\left(k^{-7}\right), \quad k \rightarrow \infty, \mu>0 ; \\
\psi_{0}^{(1)}(k) \sim k^{-1}[1-r\{\cosh (\pi r)+\sin (2 k)\} / k \sinh (\pi r)], \quad \\
\psi_{0}^{(2)}(k) \sim \cos (2 k) / k \pi,
\end{gathered}
$$

Evidently, the response first rises proportional to $k$ and then falls with some negative power of $k$. We now compare this behaviour with that obtained by a primitive perturbation series and by harmonic truncation.

\subsection{Primitive perturbation series}

In this case one regards the advection term $-i k \hat{\eta} \sin t$ in the lefthand side of (30) as a small perturbation as one would do in the case of a fully nonlinear problem. It is also the approach followed by Huthnance (1973) for a finite amplitude topography. The solution for the residual vorticity field has already been given by Zimmerman (1980), and reads

$$
\hat{\eta}_{0}^{(1)} / f=\hat{\eta}_{0}^{(2)}=\frac{1}{2} i k a(k) /\left[1+b^{2}(k)\right] .
$$

As $b(k)=r+\mu k^{2}$, one recovers the asymptotic regime of $\hat{\eta}_{0}^{(i)}$ for $k \rightarrow 0$, as given by (40), (42) and (44). However for $k \rightarrow \infty$ we now have, for $\mu=0$,

$$
\hat{\eta}_{0}^{(i)} / a(k) \sim k,
$$

in contrast to (47) and (48). This is the "ultraviolet catastrophe" mentioned in the introduction, making this approach invalid for large wavenumbers.

\subsection{Harmonic truncation}

Another approach to solve (30), used by Loder (1980) and further discussed by Young (1983), is to write the solution for $\hat{\eta}$ first as a 
harmonic series

$$
\hat{\eta}=\hat{\eta}_{0}+\hat{\eta}_{1} e^{i t}+\hat{\eta}_{2} e^{2 i t}+\cdots
$$

and then to truncate the series at same order $n$, in which case (30) leads to a set of $n+1$ coupled linear equations. As one is usually interested in the residual vorticity $\hat{\eta}_{0}$, the lowest non-trivial truncation is

$$
\hat{\eta}=\hat{\eta}_{0}+\hat{\eta}_{1} e^{i t}
$$

Substituting (52) in (30), neglecting terms proportional to $e^{2 i t}$, one then finds for the residual vorticity, again subdivided in $\hat{\eta}_{0}^{(1)}$ and $\hat{\eta}_{0}^{(2)}$

$$
\hat{\eta}_{0}^{(1)} / f=\hat{\eta}_{0}^{(2)}=\frac{1}{2} i k a(k) /\left[1+b^{2}(k)+\frac{1}{2} k^{2}\right] .
$$

As can be seen now both for $k \rightarrow 0$ and $k \rightarrow \infty(\mu=0)$ the asymptotic behaviour of (53) is similar to that of (40), (42) combined with (44), (47) and (48), apart from the oscillating character of (47) and (48). This is obviously an important improvement compared with the results of a primitive perturbation series.

In order to obtain a fair judgement of the approximations, compared with the exact solution, we give in Figure 1 the various weighting functions both for $r=0$ and $r=1(\mu=0)$. (The weighting functions in (49) and (53) are obtained by dividing through $i a(k)$.) The asymptotic arguments for $k \rightarrow 0$ of all solutions is obvious, as is the failure of the primitive perturbation series for $k \rightarrow \infty$. However, the comparison of the approximate solutions by harmonic truncation and the exact solution for $k=O(1)$ and larger, leads to different conclusions depending on the specific vorticity production mechanism and on the value of the damping parameter $r$. Evidently, the agreement is better for the residual vorticity derived from topographic planetary vortex stretching, the more so if frictional damping is stronger; it is nearly perfect for $r=1$. On the other hand, the results are less satisfactory for residual vorticity derived from differential bottom friction, even though the asymptotic behaviour for $k \rightarrow \infty$ is in agreement. In the next chapter we shall show that the exact shape of the residual current velocity profile for a specific shape of the bottom topography may show large qualitative devia- 

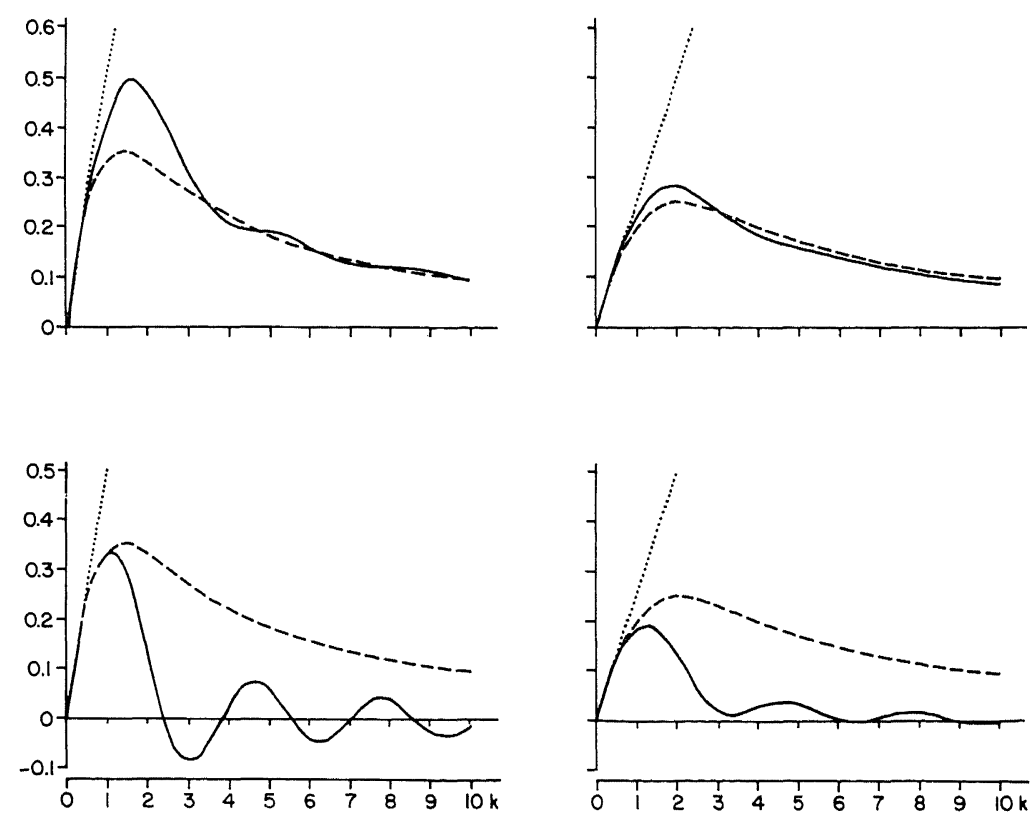

Figure 1 Residual vorticity response functions for planetary vortex stretching (upper diagrams) and differential bottom friction (lower diagrams) both for $r=0$ (left) and $r=1$ (right), with $\mu=0$. The drawn curves are the exact results given by (41) and (43). The broken curves give the results by harmonic truncation (53). The dotted lines are the results by a primitive perturbation series, (49).

tions from those derived by using the harmonically truncated form of the weighting functions, even in the case (Figure 1b) where the agreement between exact and approximated weighting functions seems to be nearly perfect.

\section{FOURIER BACK-TRANSFORMATION FOR A STEP PROFILE}

Having obtained the exact solution of $(30)$ for $\hat{\eta}(k, t)$, we obtain the vorticity field $\eta(x, t)$ in physical space by applying (22), assuming $\hat{h}(k)$ to be known. We shall discuss the back-transformation for a specific one-dimensional profile that approaches the Heaviside step function 
if a parameter approaches infinity. The relevance of such a profile is evident from the work of Loder (1980) on residual currents at a shelf break. Let $h(x)$ be given as

$$
h(x)=h_{*} \tan ^{-1}(\beta x),
$$

where $h_{*}$ is the dimensionless step height. If $\beta \rightarrow \infty, h(x)$ becomes the step function. Evidently

$$
\partial h / \partial x=\beta h_{*} /\left(\beta^{2} x^{2}+1\right)
$$

so that

$$
a(k)=-i k \hat{h}(k)=\frac{1}{2} h_{*} e^{-k / \beta} .
$$

From here on our interest is mainly in the shape of the residual velocity profile $v(x)$ over the step, rather than the residual vorticity. Dropping the 0 subscripts, the relationship between $v$ and $\eta$ is simply

$$
\eta^{(j)}=\partial v^{(j)} / \partial x
$$

hence

$$
\hat{v}^{(j)}(k)=i \hat{\eta}^{(j)}(k) / k
$$

where $\hat{\eta}(k)$ is given by (40) and (42). The superscript has the same meaning as in (41), (43). Thus after back-transformation the residual velocity profile of the current along the isobaths is

$$
\begin{aligned}
v^{(j)}(x) & =-c_{j} \int_{-\infty}^{\infty} a(k) k^{-1} \psi_{0}^{(j)}(k) e^{-i k x} d k \\
& =-\left(c_{j} / 2 \pi\right) \int_{-\infty}^{\infty} h^{\prime}(\zeta) F^{(j)}(x-\zeta) d \zeta
\end{aligned}
$$

where

$$
F^{(j)}(x)=\int_{-\infty}^{\infty} k^{-1} \psi_{0}^{(j)}(k) e^{-i k x} d k
$$

and

$$
c_{1}=f, \quad c_{2}=r .
$$


Zimmerman (1981b) discussed two limits of (60) for $\beta \rightarrow 0$ and $\beta \rightarrow \infty$. It can easily be seen that for $\beta \rightarrow 0$ (up to a multiplication constant)

$$
v^{(j)}(x) \sim-\beta h_{*} /\left(\beta^{2} x^{2}+1\right)=-\partial h / \partial x
$$

which follows from (60) and the asymptotic behaviour of $k^{-1} \psi_{0}^{(j)}(k)$ for $k \rightarrow 0$. In this limit, which physically means that the tidal excursion is much smaller than the stepwidth, the residual velocity profile is simply the mirror image of the bottom slope. More interesting is the other extremum, $\beta \rightarrow \infty$, which means a stepwidth much smaller than the tidal excursion. In that case

$$
\partial h / \partial x \rightarrow \pi h_{*} \delta(x), \quad a(k) \rightarrow \frac{1}{2} h_{*}
$$

hence

$$
v^{(j)}(x)=-\frac{1}{2} c_{j} h_{*} F^{(j)}(x)
$$

Therefore, in this case, we have to deal with the Fourier backtransform defined by (61). This is the subject of the next sections.

\subsection{Quasi-Lagrangian residual velocity profile}

As in Section 3 we solve for the Eulerian residual velocity field after first having obtained the quasi-Lagrangian residual velocity profile at the step. Analogously to (58)-(59), using (37)-(39), and taking the limit $\lambda \rightarrow \infty$ the quasi-Lagrangian residual velocity denoted by $w(x)$ is given by

$$
w^{(j)}(x)=\frac{1}{2} i h_{*} c_{j} \int_{-\infty}^{\infty} k^{-1} \phi_{0}^{(j)}(k) e^{-i k x} d k
$$

As from (37), $\phi_{0}^{(1)}=0$, we are left with

$$
w^{(2)}(x)=\frac{1}{2} h_{*} r \int_{-\infty}^{\infty}\left[J_{0}^{\prime}(k) / k b(k)\right] e^{i k x} d k
$$

which for $b(k)=r(\mu=0)$ reads

$$
w^{(2)}(x)=-h_{*} \int_{0}^{\infty} J_{1}(k) k^{-1} \cos k x d k
$$


or

$$
w^{(2)}(x)= \begin{cases}-h_{*}\left(1-x^{2}\right)^{1 / 2}, & |x| \leqq 1 \\ 0, & |x|>1\end{cases}
$$

Evidently, by excluding vorticity diffusion $(\mu=0)$ the quasiLagrangian residual velocity field has a finite width, which for a step topography is exactly equal to the tidal excursion. We shall see that this structure is also present in the Eulerian residual velocity field. For $\mu \neq 0$ vorticity diffusion evenly spreads the velocity profile, which for $|x|>1$ can then be shown to read (Temme and Zimmerman, 1985)

$$
w^{(2)}(x)=-\frac{1}{2} \pi h_{*} \exp \left[-|x| r^{1 / 2} \mu^{-1 / 2}\right] I_{1}\left(r^{1 / 2} \mu^{-1 / 2}\right), \quad x \geqq 1,
$$

where $I_{1}(z)$ is the modified Bessel function of the first kind, order 1 .

\subsection{Eulerian residual velocity field at a step}

In the Appendix we evaluate the Fourier transforms of $\psi_{0}^{(j)}(k) / k$ for the special case $\mu=0$, i.e. $b(k)=r=$ constant. The functions

$$
F^{(j)}(x)=\int_{-\infty}^{\infty} \psi_{0}^{(j)}(k) k^{-1} e^{i k x} d k, \quad j=1,2,
$$

are expressed in terms of hypergeometric functions. First we will show that the functions in (71) vanish identically outside the $x$ interval $(-2,2)$.

To prove this consider the integral

$$
\int_{C_{r}} \psi_{0}^{(j)}(z) z^{-1} e^{i z x} d z
$$

where $C_{r}$ consists of the following parts: $C_{r}=C_{r}^{(1)} \cup C_{r}^{(2)}$, with

$$
C_{r}^{(1)}=\{z \mid-r \leqq z \leqq r\}, \quad C_{r}^{(2)}=\left\{z \mid z=r e^{i \theta}, 0 \leqq \theta \leqq \pi\right\},
$$

and where $r$ is a positive real number. Now we use $(47,48)$ with $k$ replaced by the complex number $z \in C_{r}^{(2)}(r$ large). It follows that the function $e^{i z x}$ dominates $\left|\psi_{0}^{(j)}(z)\right|$ in the upper half plane $\operatorname{Im} z>0$ when 
$x>2$. For $x<-2$ we can use a contour in the lower half plane. Therefore, if $x>2$, the $z$-integral over $C_{r}$ (which vanishes identically since there is no singularity in the finite domain with boundary $C_{r}$ ) equals the integral over $C_{r}^{(1)}$ in the limit $r \rightarrow \infty$. Also if $x= \pm 2$ this is true, owing to

$$
\psi_{0}^{(j)}(z) z^{-1} e^{i z x}=O\left(z^{-2}\right), \quad z \in C_{r}^{(2)}
$$

So we obtain

$$
F^{(j)}(x)=0, \quad x \leqq-2 \text { or } x \geqq 2,
$$

which is twice the interval over which the Lagrangian velocity profile extends.

An analytical solution of the Eulerian velocity profiles can finally be obtained by performing the transform in (71), which includes various manipulations with the Bessel function series in (41) and (43), as well as with some relation between special functions. The details are contained in the Appendix. Here we only quote the final result, stating that (for $b=r$ )

$$
F^{(2)}(x)=\left[\pi\left(1-\frac{1}{4} x^{2}\right) / \sinh (\pi r)\right]_{2} F_{1}\left(\frac{1}{2}-i r, \frac{1}{2}+i r ; 2 ; 1-\frac{1}{4} x^{2}\right),
$$

where ${ }_{2} F_{1}$ is a Gauss hypergeometric function. $F^{(2)}(x)$ obeys (72). The other transform, $F^{(1)}(x)$, can be expressed in $F^{(2)}(x)$ as

$$
F^{(1)}(x)=F^{(1)}(0)+2 r x \int_{0}^{x} \zeta^{-2}\left[F^{(2)}(0)-F^{(2)}(\zeta)\right] d \zeta+c x,
$$

where $c$ follows from $F^{(1)}(2)=0$, as also (74) must obey (72).

Finally, in order to compare the exact residual velocity profiles with those obtained by harmonic truncation, we use (58)-(59) together with (53) to get

$v^{(1)}(x) / f=v^{(2)}(x)=-\frac{1}{4} h_{*} \int_{-\infty}^{\infty}\left[1+r^{2}+\frac{1}{2} k^{2}\right]^{-1} e^{i k x} d k=-\frac{1}{2} h_{*} \pi \Delta^{-1} e^{-|x| \Delta}$,

where

$$
\Delta=\left\{2\left(1+r^{2}\right)\right\}^{1 / 2}
$$


Figure 2a, $\mathrm{b}$ then gives, for $r=1$, the various residual current velocity profiles up to a factor $\frac{1}{2} h_{*}$ (and $f$ for $j=1$ ). Shown are the Eulerian profiles for both production mechanisms as expressed in (73) and (74), as well as those profiles obtained by harmonic truncation, expressed in (75), (76). Also drawn is the Lagrangian current velocity profile given by (69), which vanishes for production by planetary vortex stretching, and therefore is absent in Figure $2 \mathrm{~b}$.

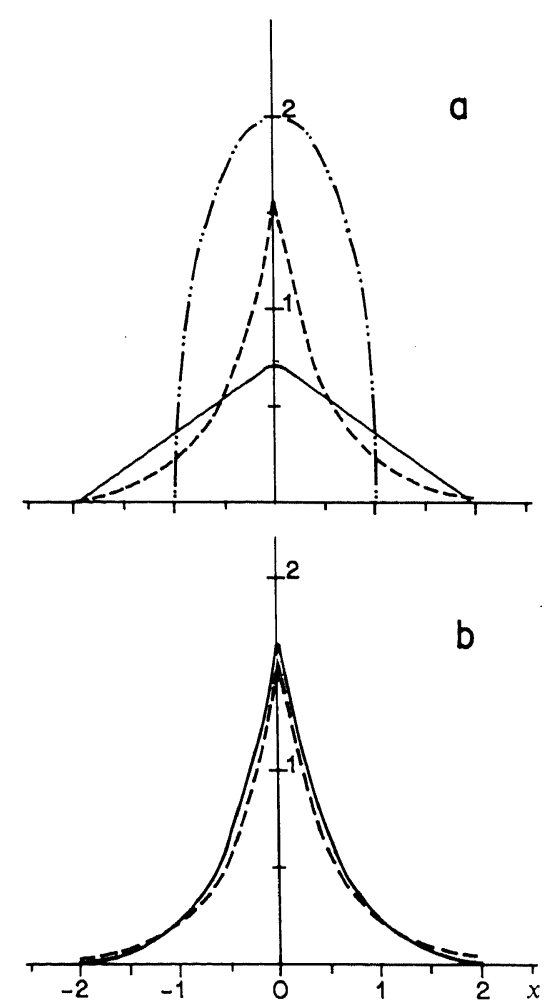

Figure 2 The residual velocity profiles at a step in the bottom topography at $x=0$. Shown are the profiles for the planetary vortex stretching mechanism (b) and for differential bottom friction (a), both for the exact solution (73), (74) (drawn curves) as well as for the result obtained by harmonic truncation, (75) (broken curves). The Lagrangian veloxity profile in (a) is shown by a dotted curve corresponding to (69). 


\section{DISCUSSION}

Inspection of Figure 2 immediately reveals an important qualitative difference between the exact Eulerian residual current velocity profiles and those obtained by the method of harmonic truncation. Whereas the former leads to velocities that vanish identically outside the interval $(-2,2)$, the latter gives an exponentially decaying profile. As to the Lagrangian profile in Figure 2a, also here we have an interval over which the residual current exists, albeit $(-1,1)$ in this case. These finite intervals of the exact solutions have an obvious physical interpretation. Vorticity is only produced over a sloping bottom, that is, at $x=0$ for a step-profile. As, for the Lagrangian profile, the coordinate $x$ is the tidal average coordinate of a water parcel that performs an oscillatory displacement with amplitude 1, parcels with mean coordinate outside the interval $(-1,1)$ will not reach the step, hence will not experience any change of their relative vorticity. The residual Lagrangian profile therefore vanishes outside that interval. The Eulerian profiles, on the other hand, describe the residual velocity field as seen by an observer at rest relative to the oscillatory tidal displacements across the step topography. The farthest a water parcel that crosses the step can get away from the step, is two times the excursion amplitude. Hence outside the interval $(-2,2)$ no change of relative vorticity is observed at any stage of the tidal period, meaning absence of residual effects there.

Evidently, the physics described above is misrepresented by using harmonic truncation as an approximation to the exact solution of the basic equation. In accordance with Figure 1, Figure 2 shows that the distortion is less serious for the residual profile generated by the planetary vortex stretching mechanism, Figure $2 b$, than it is for the profile generated by differential bottom friction, Figure 2a. In both cases, however, exponential decay of the residual velocity profile obtained by harmonic truncation exists, which is reminiscent of the behaviour that the exact solution would show if horizontal vorticity diffusion were included $(\mu \neq 0)$; see also Eq. (70). Apparently, harmonic truncation leads to spurious horizontal diffusion. It can therefore misrepresent the actual width of the residual current profile as has also been shown by Young (1983) discussing the moments of the velocity distribution.

As to the "amplitude" of the Eulerian residual current profile 


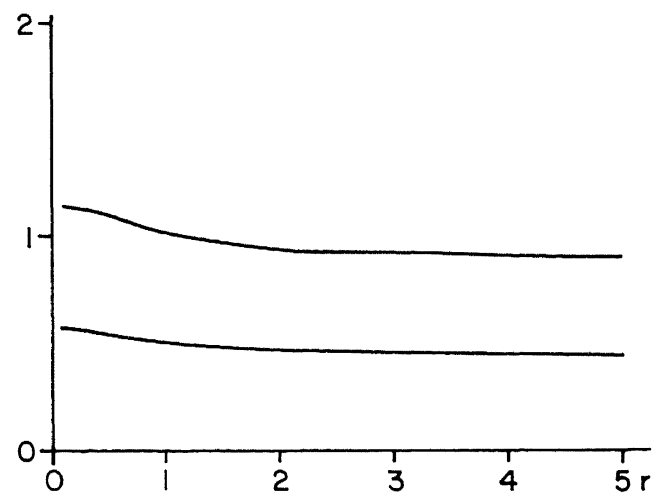

Figure 3 The ratio of the residual velocity at the step $(x=0)$ from the exact solution and from harmonic truncation is plotted as a function of the damping rate $r$. Upper curve $\Delta F^{(1)}(0) / \pi$, lower curve $\Delta r F^{(2)}(0) / \pi$.

obtained by both methods-i.e. the values of $v(0)$-we have plotted in Figure 3 the ratios $\Delta F^{(1)}(0) / \pi$ and $\Delta r F^{(2)}(0) / \pi$ as a function of the damping rate $r . F^{(1)}(0)$ is given by $(\mathrm{A} 9), F^{(2)}(0)$ by $(\mathrm{A} 12)$ and $\Delta$ by (76). As could already be expected from the results shown in Figure 1, the amplitude of the residual velocity profile generated by vortex stretching is well-produced by harmonic truncation. It is only underestimated by about $15 \%$ for weak friction $(r \rightarrow 0)$. On the other hand, that part of residual velocity profile that is produced by differential bottom friction is always overestimated by about a factor 2 as could already be expected in view of Figure 1 (lower side). All in all, harmonic truncation accords with exact results only if the planetary vortex stretching mechanism is the dominant generation mechanism of topographic vorticity.

\section{Acknowledgement}

One of us (L. R. M.) is supported by a grant from the Netherlands Organization for the Advancement of Pure Research (Z.W.O.).

\section{References}

Abramowitz, M. and Stegun, I. A., Handbook of Mathematical Functions, Natl. Bureau Standards Appl. Math. Series, 55, U.S. Government Printing Office, Washington, D.C. (1964). 
Gradshteyn, I. S. and Ryzhik, W., Tables of Integrals, Series and Products, Academic Press, New York (1965).

Huthnance, J. M., "Tidal current asymmetries over the Norfolk sandbanks", Estuar. Coastal Mar. Sci. 1, 89-99 (1973).

Huthnance, J. M., "On mass transports generated by tides and long waves", J. Fluid Mech., 102, 367-387 (1981).

Loder, J. W., Topographic rectification of tidal currents on the sides of Georges Bank," J. Phys. Oceanogr. 10, 1399-1416 (1980).

Luke, Y. L., The Special Functions and their Approximations, Academic Press, New York (1969).

Robinson, I. S., "Tidally induced residual flow," in: Physical Oceanography of Coastal and Shelf Seas (ed. B. Johns), 321-357 (1983).

Temme, N. M. and Zimmerman, J. T. F., "On the theory of topographic vorticity production by tidal currents," Centre for Math. Computer Sci., Amsterdam, Rpt AM-R8506, $20 \mathrm{pp}$ (1985).

Watson, G. N., A Treatise on the Theory of Bessel Functions, Cambridge University Press (1944).

Young, W. R., "Topographic rectification of tidal currents," J. Phys. Oceanogr. 13, 716-721 (1983).

Zimmerman, J. T. F., "Topographic generation of residual circulation by oscillatory (tidal) currents," Geophys. Astrophys. Fluid Dyn. 11, 35-47 (1978).

Zimmerman, J. T. F., "Vorticity transfer by tidal currents over an irregular topography," J. Mar. Res. 38, 601-630 (1980).

Zimmerman, J. T. F., "Dynamics, diffusion and geomorphological significance of tidal residual eddies," Nature 290, 549-555 (1981a).

Zimmerman, J. T. F., "Topographic rectification; a comment on spectral representation," J. Phys. Oceanogr. 11, 1037-1039 (1981b).

\section{Appendix}

\section{SUMMING A SERIES OF BESSEL FUNCTIONS}

To give an explicit summation for the series in (41), (43), we start with the Parseval relation for (35), i.e.

$$
\sum_{m=-\infty}^{\infty} J_{m}^{2}(k)=1
$$

(Watson, 1944, p. 31). It follows that

$$
\psi_{0}^{(1)}(k)=k^{-1}\left[1-J_{0}^{2}(k)\right]-2 b^{2} k^{-1} \sum_{m=1}^{\infty}\left(b^{2}+m^{2}\right)^{-1} J_{m}^{2}(k) .
$$


The remaining series follows from Luke (1969, Vol. 2, p. 52); we write it in the form

$$
\sum_{m=1}^{\infty}\left(b^{2}+m^{2}\right)^{-1} J_{m}^{2}(k)=[\pi / 2 b \sinh (\pi b)] J_{i b}(k) J_{-i b}(k)-\frac{1}{2} b^{-2} J_{0}^{2}(k) .
$$

Hence, for $\psi_{0}^{(1)}(k)$ we obtain

$$
\psi_{0}^{(1)}(k)=k^{-1}\left\{1-[\pi b / \sinh (\pi b)] J_{-i b}(k) J_{i b}(k)\right\} .
$$

The series for $\psi_{0}^{(2)}(k)$ in (43) is summed upon differentiating (A2) with respect to $k$. The result is

$$
\psi_{0}^{(2)}(k)=-[\pi / 2 \sinh (\pi b)] d\left[J_{-i b}(k) J_{i b}(k)\right] / d k .
$$

\section{ELABORATION OF $F^{(2)}(x)$}

Since $\psi_{0}^{(j)}(k, b) / k$ are even functions of $k$, the Fourier transform (71) is a cosine transform. Using (A3) we obtain

$$
d F^{(2)}(x) / d x=-[\pi x / \sinh (\pi b)] \int_{0}^{\infty} J_{i b}(k) J_{-i b}(k) \cos k x d k .
$$

From Gradshteyn and Ryzhik (1965, p. 732) we have

$$
d F^{(2)}(x) / d x=-[\pi x / 2 \sinh (\pi b)] P_{i b-1 / 2}\left(\frac{1}{2} x^{2}-1\right) .
$$

Using the relation

$$
\int_{1}^{z} P_{v}(t) d t=-\left(1-z^{2}\right)^{1 / 2} P_{v}^{-1}(z), \quad z \in(-1,1),
$$

we can integrate the equation for $d F^{(2)}(x) / d x$ into

$$
F^{(2)}(x)=[\pi / 2 \sinh (\pi b)]\left[x^{2}-\frac{1}{4} x^{4}\right]^{1 / 2} P_{i b-1 / 2}^{-1}\left(\frac{1}{2} x^{2}-1\right) .
$$

The Legendre function is associated with the so-called conical functions. It is real, although one of the parameters is complex. For 
numerical computations it is convenient to have a representation in the form of a series. This can be obtained by writing the Legendre function as a hypergeometric function (Gradshteyn and Ryzhik, 1965, p. 999):

$$
F^{(2)}(x)=\left[\pi\left(1-\frac{1}{4} x^{2}\right) / \sinh (\pi b)\right]_{2} F_{1}\left(\frac{1}{2}-i b, \frac{1}{2}+i b ; 2 ; 1-\frac{1}{4} x^{2}\right) .
$$

With the familiar expansion of the ${ }_{2} F_{1}$-function we can write this as

$$
F^{(2)}(x)=\frac{\pi\left(1-\frac{1}{4} x^{2}\right)}{\sinh (\pi b)} \sum_{n=0}^{\infty} \frac{\left(\frac{1}{2}-i b\right)_{n}\left(\frac{1}{2}+i b\right)_{n}}{(2)_{n} n !}\left(1-\frac{1}{4} x^{2}\right)^{n},
$$

where $(\alpha)_{n}=\Gamma(\alpha+n) / \Gamma(\alpha), n=0,1, \ldots$ This series is useful for values of $x$ near \pm 2 , but it converges for all $x \in[-2,2]$. For $x=0$ we have by using

$$
{ }_{2} F_{1}(a, b ; c ; 1)=\Gamma(c) \Gamma(c-a-b) / \Gamma(c-a) \Gamma(c-b), \quad \operatorname{Re}(c-a-b)>0,
$$

the value of $F^{(2)}(0)$, i.e.

$$
\left.F^{(2)}(0)=4 \operatorname{coth}(\pi b) / 4 b^{2}+1\right)
$$

From (A6) we infer that $\lim _{|x| \uparrow 2} F^{(2)}(x)=0$, so $F^{(2)}(x)$ is continuous at $x= \pm 2$.

For $x \rightarrow 0$ we can use a transformation for the hypergeometric function (Abromowitz and Stegun, 1964, p. 559, formula 15.3.11), which gives in our case

$$
\begin{aligned}
& F^{(2)}(x)=F^{(2)}(0)+\frac{1}{4} x^{2} \operatorname{coth}(\pi b) \sum_{n=0}^{\infty}\left[\left(\frac{3}{2}+i b\right)_{n}\left(\frac{3}{2}-i b\right)_{n} / n !(n+1) !\right]\left(\frac{1}{4} x^{2}\right)^{n} \\
& \quad \times\left[\ln \frac{1}{4} x^{2}-\psi(n+1)-\psi(n+2)+\psi\left(\frac{3}{2}+n+i b\right)+\psi\left(\frac{3}{2}+n-i b\right)\right] .
\end{aligned}
$$

If $\psi\left(\frac{3}{2}+i b\right)+\psi\left(\frac{3}{2}-i b\right)$ is available, then the remaining terms are easily computed by recursion.

Representations (A6) and (A8) are convenient starting points for numerical evaluation of $F^{(2)}(x)$. 


\section{ELABORATION OF $F^{(1)}(x)$}

The function $F^{(1)}(x)$ cannot be expressed in terms of a single Legendre function or a Gauss hypergeometric function, as was possible in the previous case. We express $F^{(1)}(x)$ in terms of an integral of $F^{(2)}(x)$, from which representation numerical evaluation is easily performed.

By using the relation in (A3), (A4) we obtain

$$
x d^{2} F^{(1)}(x) / d x^{2}=-2 b d F^{(2)} / d x .
$$

Hence

$$
-2 b F^{(2)}(x)=x d F^{(1)}(x) / d x-F^{(1)}(x)+F^{(1)}(0)-2 b F^{(2)}(0),
$$

where we use $\lim _{x \rightarrow 0^{+}}\left[x d F^{(1)}(x) / d x\right]=0$; note that $F^{(j)}(x)$ is even in $x$.

The value $F^{(2)}(0)$ is given in (A7). The value of $F^{(1)}(0)$ follows from (41), (71), giving

$$
F^{(1)}(0)=4 \sum_{m=1}^{\infty}\left[m^{2} /\left(b^{2}+m^{2}\right)\right] \int_{0}^{\infty} k^{-2} J_{m}^{2}(k) d k .
$$

The integrals are special cases of the Weber-Schafheitlin integrals. From Gradshteyn and Ryzhik (1965, p. 692) we obtain

$$
F^{(1)}(0)=4 \pi^{-1} \sum_{m=1}^{\infty} m^{2}\left(b^{2}+m^{2}\right)^{-1}\left(m^{2}-\frac{1}{4}\right)^{-1}=8 b\left(1+4 b^{2}\right)^{-1} \operatorname{coth}(\pi b) .
$$

It follows that the constant $F^{(1)}(0)-2 b F^{(2)}(0)$ in (A9) equals zero, so we are left with the equation

$$
d\left[x^{-1} F^{(1)}(x)\right] / d x=-2 b x^{-2} F^{(2)}(x) .
$$

Integration gives

$$
F^{(1)}(x)=2 b x \int_{x}^{2} \xi^{-2} F^{(2)}(\xi) d \xi
$$


which is the desired relation between $F^{(1)}(x)$ and $F^{(2)}(x)$. If we integrate (A11) with initial value $x=0$, we first write it as

$$
d\left\{x^{-1}\left[F^{(1)}(x)-F^{(1)}(0)\right]\right\} / d x=-2 b x^{-2}\left[F^{(2)}(x)-F^{(2)}(0)\right],
$$

which gives as a final result

$$
F^{(1)}(x)=F^{(1)}(0)+2 b x \int_{0}^{x} \xi^{-2}\left[F^{(2)}(0)-F^{(2)}(\xi)\right] d \xi+c x,
$$

where $c$ follows from $F^{(1)}(2)=0$. 